\title{
Optical study of strained double Ge/Si quantum dot layers
}

\author{
N. M. Santos ${ }^{1}$, B. P. Falcão ${ }^{1}$, J. P. Leitão ${ }^{1}$, N. A. Sobolev ${ }^{1}$, M. C. \\ Carmo $^{1}$, N. S. Stepina ${ }^{2}$, A. Yakimov ${ }^{2}$ and A. I. Nikiforov ${ }^{2}$ \\ ${ }^{1}$ Departamento de Física and I3N, Universidade de Aveiro, 3810-193 Aveiro, Portugal \\ ${ }^{2}$ Rzhnov Institute of Semiconductor Physics, Siberian Branch of the Russian Academy of \\ Sciences, 630090 Novosibirsk, Russia \\ E-mail: joaquim.leitao@ua.pt
}

\begin{abstract}
In this work we studied experimentally and theoretically the emission from Ge WL and QDs. The numerical calculations give a prediction for the energy positions of the WL and QDs related emissions in accordance with the PL measurements. The experimental results show an independence of the energy position of the WL related emission of the interaction between the two deposited Ge layers whereas a shift to higher energies was observed for the dots related emission with the increase of the Si spacer thickness. Two different transitions (A and B) related to QDs were identified. The temperature dependence of the intensity was investigated. No dependence of the activation energies on the Si spacer thickness was observed.
\end{abstract}

\section{Introduction}

Vertically aligned Ge quantum dots (QDs) are an example of a system that explores the elastic strains arising from the buried islands along the structure as a driving force for the self-assembling of islands at the top of the grown islands [1]. In strained Ge/Si heterostructures, charge carriers are localized on opposite sides of the interface between both materials. The electron energy levels can be significantly modified by the elastic deformation in both dots and neighborhood [2]. Double-dot systems are the simplest examples of "artificial molecules" with applications in quantum information processing [3, 4]. For this system the interaction between the dots can be varied in a controlled way through the change of the thickness of the Si spacer layer between the Ge layers.

In the present work we carried out the study by means of photoluminescence (PL) measurements of samples containing two layers of 5 monolayers (ML) of Ge separated by a $\mathrm{Si}$ spacer layer with varying thickness in the range $20-40 \AA$. Another sample with just one Ge layer was considered as reference. The influence of the Si spacer thickness on the SiGe wetting layer (WL) and Ge quantum dots (QDs) related emissions as well as the temperature dependence of the PL are discussed.

\section{Experimental details}

Five samples with two Ge layers separated by a Si spacer layer were grown at $500^{\circ} \mathrm{C}$ by molecular beam epitaxy (MBE) on a Si (001) substrates. The nominal thickness of the Ge layers was equal to $5 \mathrm{ML}$ whereas the thickness $(d)$ of the Si spacer was varied in the range from $20-40 \AA$ (see 
figure 1). Additionally, a reference sample with just one Ge layer was also studied. After the growth, some pieces of the samples were passivated with atomic hydrogen in a CVD reactor at $T \sim 100^{\circ} \mathrm{C}$ for $30 \mathrm{~min}$, in order to decrease the density of recombination active defects.

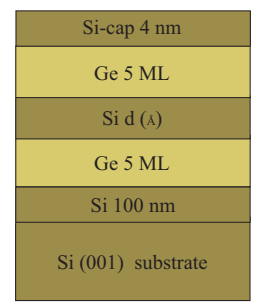

Figure 1. Schematic drawing of the set of samples studied. Sample \# 1 has only one Ge layer. The spacer thicknesses $d$ for the other samples are: $20 \AA$ (\#2); $25 \AA$ (\#3); $30 \AA$ (\#4); $35 \AA$ (\#5); $40 \AA$ (\#6).

PL was performed for all samples, in the range from $5-300 \mathrm{~K}$, using a FTIR Bruker IFS 66v spectrometer equipped with a Ge detector cooled to liquid nitrogen temperature. The $488 \mathrm{~nm}$ line of an $\mathrm{Ar}^{+}$laser was used as excitation source. The temperature of the sample was changed in the range from $4-300 \mathrm{~K}$.

\section{Results and discussion}

In figure 2 we show the PL spectra of as grown and passivated samples. For the as grown samples we observe a similar emission for all samples with a superposition of sharp transitions with a broad band. A few of the sharp lines correspond to the recombination of bound excitons in Si. The broad band and the majority of the sharp transitions are related to defects because after the atomic hydrogen passivation they disappear. As is well known, the passivation treatment reduces strongly the number of recombination active defects diminishing the competition for charge carriers. As a result, we observe a dramatic change of the PL spectra for all samples (see figure $2 \mathrm{~b}$ )). At energies just below the energy gap of $\mathrm{Si}$, we observe the usual emission from free and bound excitons in Si. At lower energies, the broad band with maximum of intensity at $\sim 1.045 \mathrm{eV}$ is attributed to the SiGe WL. In the range $h \nu<1.0 \mathrm{eV}$, we observed two bands ascribed to transitions related to the Ge QDs. In a previous study on the samples used in this work, cross-section transmission electron microscopy revealed the presence of QDs in each Ge layer $[6]$.
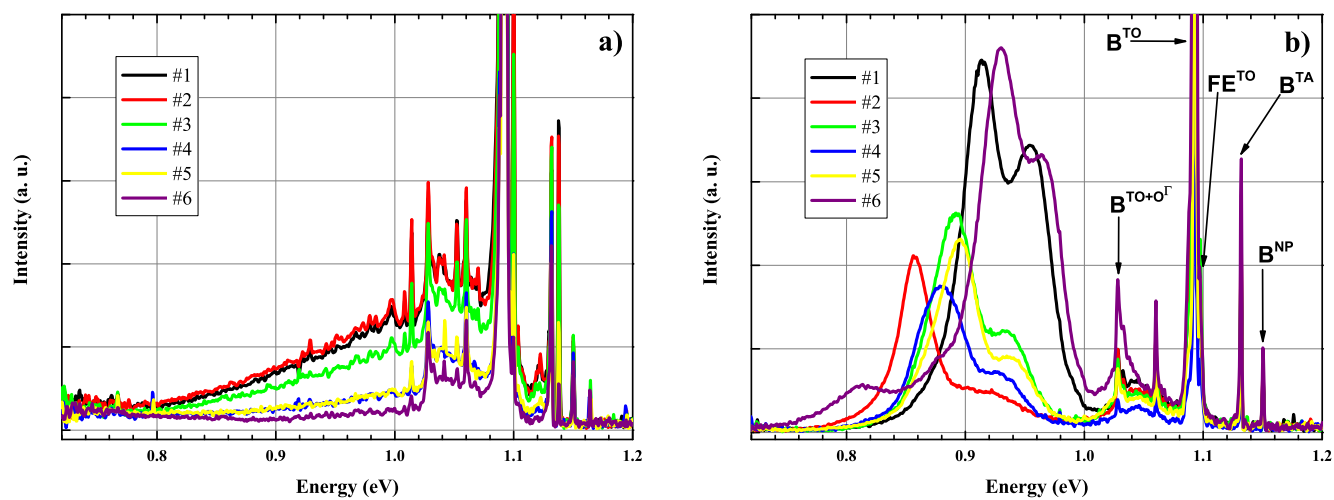

Figure 2. a) PL spectra of the as grown samples at $5 \mathrm{~K}$; b) PL spectra of the passivated samples at $5 \mathrm{~K}$; the free and bound excitons related emissions are identified in the spectra.

The assignment of the optical transitions is supported by numerical calculations [5]. A sixband $\mathbf{k} \cdot \mathbf{p}$ formalism was used to study the $\Gamma_{25^{\prime}}$ hole states and single-band approach to obtain the 
$\Delta_{1}$ electron state interacting with hole. The elastic strain due to the lattice mismatch between Ge and Si was included into the problem via Bir-Pikus Hamiltonian. Typical pyramidal Ge/Si islands with four $\{105\}$-oriented facets and a (001) base, lying on a 4 ML SiGe WL, have been under study. The pyramid base length $l$ is $10 \mathrm{~nm}$, the pyramid aspect ratio $h / l$ is fixed and equal to 0.1. The ground-state transition energies for the QD and WL were found to be $850 \mathrm{meV}$ and $1030 \mathrm{meV}$, respectively, in accordance with the PL measurements.

The Ge WL related emission does not shift with the variation of the Si spacer thickness, suggesting a similar Ge content and a constant thickness of the WL. However, the QDs related emission depends critically on the thickness of the Si spacer. In order to describe this dependence, the dots related emission was fitted with two gaussian curves (A, B) as ilustrated in figure 3 a). An additional gaussian curve was used at lower energies and was related to defects. The peak positions of the components $\mathrm{A}$ and $\mathrm{B}$, obtained for all samples, are presented in table 1 . Comparing the PL spectra of samples \#1 (deposition of one Ge layer) and \#2 $(d=20 \AA$ ) we observe a shift of the QDs related emission to lower energies by $\sim 57 \mathrm{meV}$. With the increasing Si spacer thickness the QDs related emission shifts to higher energies. For the sample with the thickest spacer studied (\#6, $d=40 \AA$ ), the peak positions are very close to the ones observed for the sample \#1. The influence of the Si spacer thickness on the energy of the QDs related emission is undoubtedly observed.
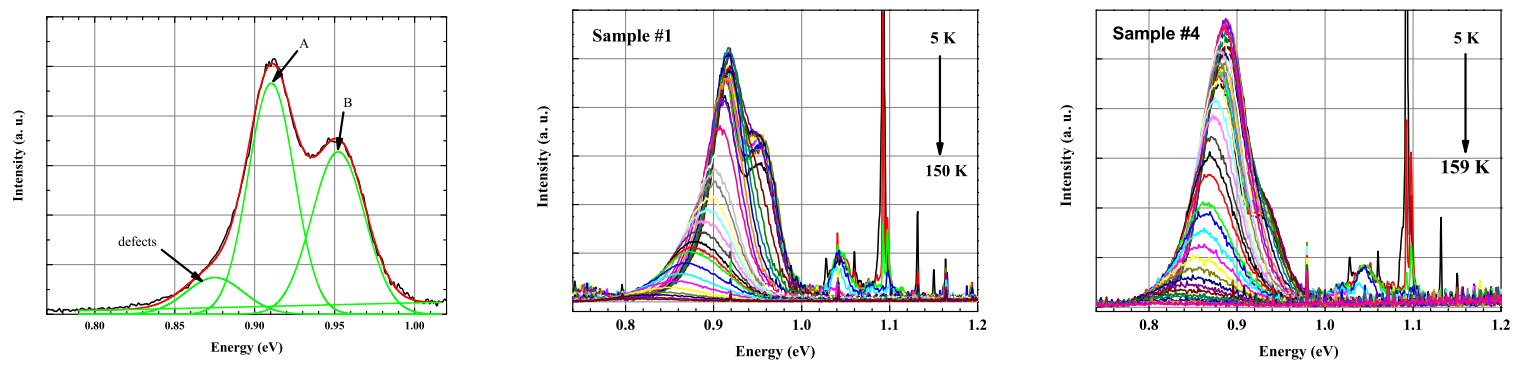

Figure 3. a) Fit with two gaussian curves (A and B) to the emission related to Ge QDs measured at $5 \mathrm{~K}$. Temperature dependence of the Ge QDs for samples: b) \#1 and c) \#4. The excitation power was $\sim 15 \mathrm{~mW}$.

Table 1. Peaks positions for the gaussian curves (A and B) used for the fit of the two gaussian curves to the Ge QDs related emission.

\begin{tabular}{lcccccc}
\hline & $\# 1$ & $\# 2$ & $\# 3$ & $\# 4$ & $\# 5$ & $\# 6$ \\
\hline component A (meV) & 0.910 & 0.857 & 0.890 & 0.879 & 0.895 & 0.928 \\
component B (meV) & 0.952 & 0.900 & 0.939 & 0.933 & 0.942 & 0.968 \\
\hline
\end{tabular}

The temperature dependence of the QDs related emission for samples \#1 and \#4 are shown in figure $3 \mathrm{~b}$ ) and c). We observe that for both samples, the component $\mathrm{B}$ decreases more rapidly than component $\mathrm{A}$. With increasing temperature the peak positions of both components do not shift in energy for $T \lesssim 80 \mathrm{~K}$, after which they shift to lower energies. The Arrhenius plot for the intensities of components $\mathrm{A}$ and $\mathrm{B}$ for samples \#1 and \#4 are shown in figure 4. For the two components in both samples we observe a raise of the intensity until $\sim 70 \mathrm{~K}$ followed 

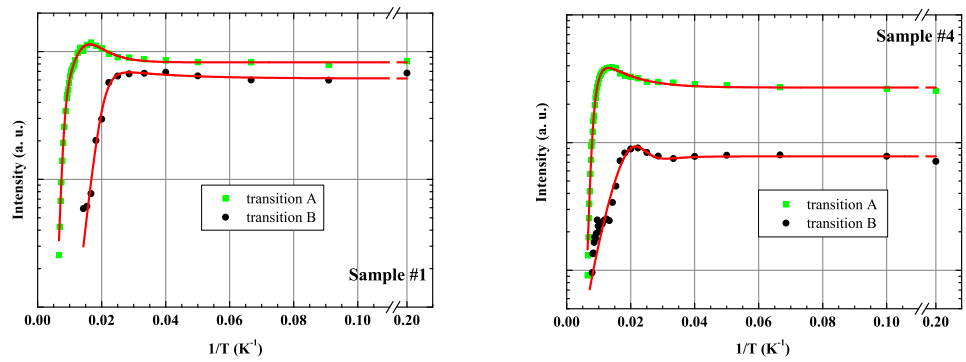

Figure 4. Arrhenius plots of the PL intensity vs. temperature for samples: a) \#1; b) $\# 4$.

by a quenching of the emission at higher temperatures. In order to describe the non radiative mechanisms responsible for the extinction of the luminescence several models were tested. The best fit was obtained considering for component B the existence of one discrete level and one band with activation energies of $E_{1}$ and $E_{2}$ [7]. For component A the fit was ontained with just the mechanism involving the band. The raise of the intensity was parameterized by an external center with an activation energy $E_{3}$, responsible for the supply of excitons/charge carriers. The fitted curves [7] to the experimental data are shown in figure 4 and the resulting activation energies in table 2. The thermal extinction process for both components involves the activation of different non radiative mechanisms. In this way, we attribute the two components to two different transitions in the QDs. The available data do not allow the identification of the nature of these transitions. No dependence of the activation energies on the Si spacer thickness was observed.

Table 2. Activation energies obtained for samples \#1 (deposition of one Ge layer) and \#4 $(d=40 \AA)$.

\begin{tabular}{c|ccc|cc}
\hline & \multicolumn{3}{|c}{ Component B } & \multicolumn{2}{c}{ Component A } \\
sample & $E_{1} \mathrm{meV}$ & $E_{2} \mathrm{meV}$ & $E_{3} \mathrm{meV}$ & $E_{2} \mathrm{meV}$ & $E_{3} \mathrm{meV}$ \\
\hline$\# 1$ & $35 \pm 16$ & $143 \pm 30$ & $17 \pm 7$ & $41 \pm 8$ & $5 \pm 18$ \\
$\# 4$ & $61 \pm 12$ & $157 \pm 26$ & $10 \pm 4$ & $16 \pm 4$ & $36 \pm 38$ \\
\hline
\end{tabular}

\section{Acknowledgements}

This work was supported by Fundação para a Ciência e Tecnologia through the project PTDC/FIS/72843/2006. J. P. Leitão would like to acknowledge the financial support from Fundação Calouste Gulbenkian.

\section{References}

[1] Tersoff J., Teichert C. and Lagally M. G. 1996 Phys. Rev. Lett. 761675

[2] Yakimov A. I., Bloshkin A. A. and Dvurechenskii A. V. 2008 Phys. Rev. B 78165310

[3] Loss D. and DiVicenzo D. P. 1998 Phys. Rev. A 57120

[4] Hanson R. and Burkard G. 2007 Phys. Rev. Lett. 98050502

[5] Yakimov A.I., Bloshkin A. A. and Dvurechenskii A. V. 2008 Appl. Phys. Lett. 93132105

[6] Yakimov A.I., Mikhalyov G. Yu., Dvurechenskii A.V. and Nikiforov A. I. 2007 J. Appl. Phys. 102093714

[7] Sobolev N. A., Fonseca A., Leito J. P., Carmo M. C., Presting H. and Kibbel H. 2003 Phys. Stat. Sol. (c) 0 1267 